\title{
Using symmetry to design pulse sequences in solid-state NMR
}

\author{
Andreas Brinkmann, Marina Carravetta, Xin Zhao, Mattias Edén, \\ Jörn Schmedt auf der Günne, and Malcolm H. Levitt
}

\author{
Physical Chemistry Division, Stockholm University, S-10691 Stockholm, Sweden
}

\section{Introduction}

Modern solid-state NMR employs a range of rf pulse sequences for a variety of tasks. There are decoupling sequences which reinforce the averaging effect of the magic-angle rotation, causing different spin species to evolve approximately independently of each other. There are also recoupling sequences which undo the averaging effect of the magic-angle rotation, temporarily restoring couplings which are otherwise inactivated by the sample spinning. The success of solid-state NMR in biological research may depend on the development of decoupling and recoupling pulse sequences which are robust with respect to a variety of undesirable spin interactions and experimental imperfections, and which function over a wide range of static magnetic fields and/or spinning frequencies.

With this in mind, our group has developed a general pulse sequence design strategy which is applicable to a wide range of methodological situations. The strategy is based on the use of symmetry arguments to design rotor-synchronized pulse sequences[1-7]. The basic decoupling and recoupling properties of a certain types of pulse sequence may be predicted using the values of just three integers, called symmetry numbers. These results apply even when the spinning frequency is comparable to the nutation frequency of the spins in the rf field. Furthermore, the symmetry theorems may be inverted: It is possible to find the combinations of symmetry numbers that give rise to a set of desirable pulse sequence properties. The symmetry theory provides a theoretical framework which greatly simplifies the task of designing rotor-synchronized pulse sequences. In this short article, we sketch the basic symmetry arguments and illustrate a few of the pulse sequences we have designed and demonstrated. In addition, we show that the symmetry theorems provide insight into the behaviour of a number of existing pulse sequences. Much of this work is of a preliminary nature and will be described in more detail in forthcoming publications. 
Table 1. Classification of spin interactions in a homonuclear system. The interactions are: Isotropic chemical shift; J-coupling; chemical shift anisotropy; direct dipole-dipole coupling.

\begin{tabular}{|c|c|c|c|}
\cline { 2 - 4 } \multicolumn{1}{c|}{} & Space Rank $l$ & Spin Rank $\lambda$ & Field Rank \\
\hline Iso-CS & 0 & 1 & 1 \\
\hline J & 0 & 0 & 0 \\
\hline CSA & 2 & 1 & 1 \\
\hline DD & 2 & 2 & 0 \\
\hline
\end{tabular}

\section{Theory}

The symmetry theorems are based on the rotation properties of the spin interaction terms. Table 1 summarizes the rotation properties of the most important nuclear spin interactions for homonuclear coupled systems (we have also generalized our results to heteronuclear systems, but this will not be discussed here). Each interaction term behaves as a component of an irreducible spherical tensor with respect to rotations of either the molecules ("space rank", denoted $l$ ), the nuclear spin polarization axes ("spin rank", denoted $\lambda$ ), or the static magnetic field ("field rank"). In practice, the "field rank" is unimportant since the direction of the static field cannot normally be changed, for technical reasons. Nevertheless, the field rank is included in Table 1 for completeness. The table shows that the pairs of space-spin ranks are different for all the interactions. The symmetry theorems described below exploit this distinction by synchronized "space rotations" (i.e. bulk rotation of the whole sample) and "spin rotations" (implemented by rf field pulse sequences).

In general a spherical tensor of rank $l$ has $2 l+1$ spherical components, with indices $m=-l,-l+1 . .+l$. Similarly, each spin interaction has a set of components, with space quantum numbers $m$ and spin quantum number $\mu$ taking all possible values permitted by the space and spin ranks $l$ and $\lambda$. In the presence of a rf field, the homonuclear DD interaction has 25 components $(5 \times 5)$, while the CSA interaction has 15 components $(3 \times 5)$

The large number of rotational components contributes to the technical difficulty of the general recoupling problem. As an example, consider the task of double-quantum homonuclear recoupling, which is one of the most useful modes of reintroducing the homonuclear dipole-dipole interactions [1-9]. In this experiment, the evolution of the spin system should be rendered sensitive to only the double-quantum DD components with $\mu=-2$ and +2 . The chemical shift terms and other DD terms should be suppressed. In addition, it is often desirable that each spin component $\mu$ is only associated with a 
single space component $m$, since this leads to a favourable orientation-dependence of the recoupling (this property is called $\gamma$-encoding $[1-3,9]$ ). In order to create a $\gamma$ encoded 2Q recoupling sequence, it is therefore necessary to suppress over 30 undesirable spin interaction terms, retaining only two. Any attempt to do this by searching over the possible combinations of pulse flip angles and $\mathrm{rf}$ phases, without the assistance of symmetry, has little hope of success.

C-type sequences. So far, the symmetry theorems apply to two general classes of rotor synchronized pulse sequence. The first class is denoted $\mathrm{CN}_{\mathrm{n}}{ }^{\mathrm{v}}$, where $\mathrm{N}, \mathrm{n}$ and $\mathrm{v}$ are the three symmetry numbers $[4,6,7]$. This class of pulse sequence may be constructed as follows: (i) take $\mathrm{n}$ sample revolution periods; (ii) divide this into $\mathrm{N}$ equal intervals; (iii) fill each interval with a rf pulse sequence $\mathrm{C}$, which is a cycle, meaning that the rf fields bring the spins back to their starting states, if all other interactions are ignored; (iv) shift the phase of the qth $\mathrm{C}$ element by the angle $2 \pi \mathrm{qv} / \mathrm{N}$, where $\mathrm{q}=0,1 . . \mathrm{N}-1$. The symmetry numbers $\mathrm{n}$ and $\mathrm{v}$ are called the space and spin winding numbers [6].

The selection rule for $\mathrm{CN}_{\mathrm{n}}{ }^{\mathrm{v}}$ symmetry may be denoted $[4,6]$

$$
\bar{H}_{\operatorname{lm} \lambda \mu}^{(1)}=0 \text { if } \mathrm{mn}-\mu \nu \neq \mathrm{NZ}
$$

where $\mathrm{Z}$ is any integer (including zero). The left-hand side of this equation expresses the first-order average Hamiltonian for the term with space rank 1 , spin rank $\lambda$ and component indices $\mathrm{m}$ and $\mu$. If a term with a certain combination of quantum numbers $\operatorname{lm} \lambda \mu$ vanishes, then the pulse sequence is expected to be insensitive to this term, to a good approximation. By choosing values of $\mathrm{N}, \mathrm{n}$ and $v$ that cause Eq.(1) to be satisfied for all the undesirable terms $\operatorname{lm} \lambda \mu$, while ensuring that Eq.(1) is not satisfied for all the desirable terms, one may focus on pulse sequences which are guaranteed to be good candidates for the task in hand. 
Fig. 1 Space-spin selection diagram for $C 7_{2}{ }^{1}$. The mirror image pathways with $m=-1$ and $m=-2$ have been suppressed for the sake of clarity.

Space-spin selection diagrams [6] are useful for visualizing the consequences of Eq.(1). One example is shown for the symmetry $\mathrm{C}_{2}{ }_{2}^{1}$ in Fig. 1. For each interaction, the composition of the term mn- $\mu \mathrm{v}$ is broken into two stages, so as to make clear how the symmetry numbers $n$ and $v$ interact. The inequality in Eq.(1) is visualized by a barrier with holes separated by $\mathrm{N}$ units. Only interactions which "pass through the holes" do not satisfy the inequality in Eq.(1) and are retained in the average Hamiltonian. Interactions which "run into the wall" are suppressed in the average Hamiltonian. The diagram in Fig. 1 illustrates that the symmetry $\mathrm{C}_{2}{ }_{2}^{1}$ suppresses all terms except for the

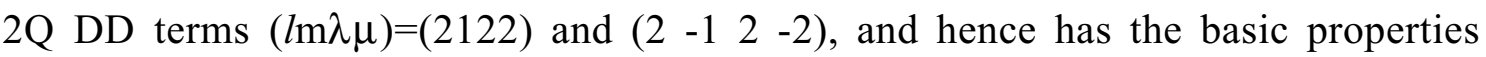
necessary for $\gamma$-encoded 2Q recoupling with chemical shift suppression. The successful C7 [1] and POST-C7 [2] sequences exploit this symmetry.

R-type sequences. The second class of symmetrical rotor-synchronized pulse sequences is based upon $180^{\circ}$ rotation elements, denoted $\mathrm{R}$, rather than cycles [7]. One construction procedure for $\mathrm{RN}_{\mathrm{n}}{ }^{\mathrm{v}}$ sequences is as follows: (i) take $\mathrm{n}$ sample revolution periods; (ii) divide this into $\mathrm{N}$ equal intervals; (iii) fill each interval with a rf pulse sequence $\mathrm{R}$, which implements a $\pi$ rotation around the $\mathrm{x}$-axis, if all other interactions are ignored; (iv) for odd-numbered elements, change the sign of all phase shifts within the element R; (v) shift the phase of the qth element by the angle $(-1)^{\mathrm{q}} \pi \mathrm{v} / \mathrm{N}$, where $\mathrm{q}=0,1 . . \mathrm{N}-1$.

This construction procedure resembles that for $\mathrm{CN}_{\mathrm{n}}{ }^{\mathrm{v}}$ sequences, except for the selection of a $180^{\circ}$ rotation element rather than a cycle, the change in the sign of the phase shift for odd-numbered elements, and the alternation of the phases implied by step (v), instead of the incrementation of the phases employed in the C-sequences.

The theory of these sequences will be described elsewhere. The selection rules for $\mathrm{RN}_{\mathrm{n}}{ }^{\mathrm{V}}$ sequences are given by [7]:

$$
\bar{H}_{l m \lambda \mu}^{(1)}=0 \text { if } m n-\mu \nu \neq(\mathrm{N} / 2) \times \mathrm{Z}_{\lambda}
$$


where $Z_{\lambda}$ is an integer of the same parity as the spin rank $\lambda$, i.e. if $\lambda$ is odd, then $Z_{\lambda}$ is an odd integer, while if if $\lambda$ is even, then $Z_{\lambda}$ is an even integer, including zero.

The selection rule (2) is more restrictive than the rule (1). In particular, the positions of the holes in the "barrier" depend on the spin rank $\lambda$ : If $\lambda$ is even, then the holes occur at levels $0, \pm \mathrm{N}, \pm 2 \mathrm{~N}$..; If $\lambda$ is odd, then the holes occur at levels $\pm N / 2, \pm 3 N / 2$.. This dependence on the spin rank $\lambda$ allows the R-sequences to discriminate between terms with the same values of $\mathrm{m}$ and $\mu$, but different values of $\lambda$, creating many new possibilities.

The scaling factor. Although the symmetry properties ensure the suppression of undesirable terms in the average Hamiltonian, they say nothing about the magnitude of the terms that are retained. In general, these terms are proportional to a scaling factor, denoted $\kappa$, that depends on the detailed structure of the elements $\mathrm{C}$ or R. Formulae for the scaling factor are given elsewhere [6]. It is normally desirable that $\kappa$ is as large as possible for the symmetry-allowed terms.

Supercycles. The performance and robustness of the symmetry-based sequences may be enhanced further by building supercycles. This is done by concatenating the original $\mathrm{CN}_{\mathrm{n}}{ }^{\mathrm{V}}$ or $\mathrm{RN}_{\mathrm{n}}{ }^{\mathrm{v}}$ sequence with one or more variant cycles, which are usually related to the original one by well-defined "mutations". Some common mutations are: (i) overall phase shifts; (ii) change in sign of the spin winding number $v$; (iii) cyclic permutation of some pulse sequence elements. The construction of supercycles must be performed with care, as it is easy to destroy some of the favourable properties of the original sequence if the supercycle construction procedure does not respect the appropriate symmetry constraints. Some examples of useful supercycles are given in refs. $[3,5,6]$, and in the discussion below. 
Fig.2. Double-quantum-filtered ${ }^{13} \mathrm{C}$ signal amplitudes in $\left[11,20-{ }^{13} \mathrm{C}_{2}\right]$-all-E-retinal, obtained using a $R 22{ }_{4}^{9}$ sequence at a static field of $9.4 \mathrm{~T}$ and a spinning frequency of $7.000 \mathrm{kHz}$. The triangles are experimental points; the solid line is a numerical simulation for a $0.299 \mathrm{~nm}$ distance, multiplied by a biexponential decay function fitted to obtain the best match with experiment (the fitting of the decay has very little impact on the estimated distance). The dashed lines show best fit simulations for distances of $0.295 \mathrm{~nm}$ and $0.304 \mathrm{~nm}$. The pulse sequence was as in ref. 1 , except that a $R 22{ }_{4}^{9}$ sequence was used instead of $C 7$, and that the $2 Q$ reconversion interval was held fixed at $5.0 \mathrm{~ms}$ while the $2 Q$ excitation interval was varied.

\section{Results and Discussion}

A brief selection of previously unpublished experimental results for some symmetrybased pulse sequences will now be given. These results are of a preliminary nature and will be published in full elsewhere.

Double-quantum homonuclear recoupling and ${ }^{13} \mathrm{C}-{ }^{13} \mathrm{C}$ distance measurements. One of the most important and challenging tasks in magic-angle-spinning NMR is to recouple homonuclear dipolar interactions, allowing the measurement of internuclear distances, for example between ${ }^{13} \mathrm{C}$ nuclei. Double-quantum schemes are particularly important in biomolecular NMR since the special phase properties of excited double-quantum coherences allow the complete suppression of signals from natural abundance ${ }^{13} \mathrm{C}$ spins, even in large molecules. The $\mathrm{C} 7$ sequence, which has the symmetry $\mathrm{C}_{2}{ }^{1}$ (see Fig.1) has been very successful and is used in a variety of guises [1-3]. However, this symmetry tends to require a rather large ratio of the rf field to the spinning frequency. 
Recently, we have experimented with different symmetries, such as $\mathrm{C} 14_{4}{ }^{5}$, which may be used in a supercycled form which requires only half the rf field of $\mathrm{C} 7$ [6]. Other groups have exploited a supercycled version of $\mathrm{C}_{2}{ }^{1}$ symmetry [5]. More recently, we have shown that a large number of promising R-sequence solutions exist [7]. These sequences are often more robust than the $\mathrm{CN}_{\mathrm{n}}{ }^{\mathrm{V}}$ sequences, especially with respect to chemical shift anisotropy. Fig. 2 shows some experimental results for a R22 ${ }^{9}$ sequence, performed on a crystalline sample of $\left[11,20-{ }^{13} \mathrm{C}_{2}\right]$-all-E-retinal in which the internuclear distance, as determined by X-ray diffraction, is $0.296 \mathrm{~nm} \mathrm{[10].} \mathrm{The}$ prominent oscillation is due to the through-space dipole-dipole coupling. The oscillation frequency provides an internuclear distance estimate of $0.299 \mathrm{~nm}$, in close agreement with the X-ray result. The precision of the distance estimate is better than $\pm 3 \mathrm{pm}$, as may be seen in Fig.2.

We have used $\mathrm{R} 14_{2}{ }^{6}$ and $\mathrm{R} 22{ }_{4}{ }^{9}$ sequences on a number of ${ }^{13} \mathrm{C}_{2}$-labelled compounds. One objective of this work has been to assess the feasibility of accurate $\mathrm{C}-\mathrm{C}$ bond length measurement in systems with large CSA. Such measurements would be very useful for elucidating the electronic structure of ligands and prosthetic groups, for example the retinylidene chromophore of rhodopsin and its relatives. So far, our results are quite encouraging. We have examined four ${ }^{13} \mathrm{C}_{2}$-labelled compounds, with the reported $\mathrm{C}-\mathrm{C}$ distances from diffraction measurements spanning the range $0.131 \mathrm{~nm}$ to $0.153 \mathrm{~nm}$ (the compounds were diammonium $\left[2,3-{ }^{13} \mathrm{C}_{2}\right]$-fumarate; ammonium hydrogen $\left[2,3-{ }^{13} \mathrm{C}_{2}\right]$ maleate; $\left[14,15-{ }^{13} \mathrm{C}_{2}\right]$-all- $E$-retinal; $\mathrm{L}-\left[2,3-{ }^{13} \mathrm{C}_{2}\right]$-alanine). This set of compounds includes both double and single $\mathrm{C}-\mathrm{C}$ bonds and spans a wide range of isotropic shifts and chemical shift anisotropies. In all cases, the ${ }^{13} \mathrm{C}-{ }^{13} \mathrm{C}$ distances determined by the $\mathrm{R} 14_{2}{ }^{6}$ sequence in a field of $9.4 \mathrm{~T}$ agreed with the diffraction methods to within $5 \mathrm{pm}$. The main source of uncertainty in the NMR distance measurement is the lack of knowledge of the CSA orientations. Despite this, the methods are sufficiently precise to allow informative measurements of $\mathrm{C}-\mathrm{C}$ bond lengths in biomolecules.

We have also measured longer distances, such as the one shown in Fig.2, as well as the $0.39 \mathrm{~nm}{ }^{13} \mathrm{C}-{ }^{13} \mathrm{C}$ distance in diammonium $\left[1,4-{ }^{13} \mathrm{C}_{2}\right]$-fumarate, which was estimated with an accuracy of $10 \mathrm{pm}$. There is more work to be done, but the method shows promise for investigating the bonding structure at the active site of large, non-crystalline biomolecules such as retinal proteins.

One point of technical importance should be noted: Although the $\mathrm{RN}_{\mathrm{n}}{ }^{\mathrm{V}}$ sequences are robust in many respects, they are exceedingly sensitive to the accuracy and stability of the rf phase shifts. We often need to adjust the rf phase in steps of $0.1^{\circ}$ in order to obtain best performance. Instrument manufacturers take note! 
Fig.3. Experimental single-quantum $2 D$ correlation spectrum of $L-\left[U_{-}{ }^{13} C\right]$-tyrosine at a field of $9.4 T$ and a spinning frequency of $23 \mathrm{kHz}$, obtained using the sequence in Eq.(3), with a duration $1 \mathrm{~ms}$.

Zero-quantum homonuclear recoupling and ${ }^{13} \mathrm{C}-{ }^{13} \mathrm{C}$ correlations. The symmetry theorems given above may readily be applied to the problem of ZQ homonuclear recoupling. Zero-quantum recoupling sequences may be used to obtain $2 \mathrm{D}{ }^{13} \mathrm{C}-{ }^{13} \mathrm{C}$ correlations maps, which are useful for spectral assignment. The symmetries may be chosen so as to provide insensitivity to isotropic and anisotropic chemical shifts, unlike the widely-used RFDR sequence, which relies on chemical shift interference in order to work at all [11]. One promising sequence uses the symmetry $\mathrm{R} 6{ }_{6}{ }^{2}$ with the basic element $\mathrm{R}=90_{180} 270_{0}$, incorporated in a 6-fold supercycle. The full sequence has the form

$$
\left[\mathrm{R}_{6}{ }^{2} \mathrm{R}_{6}{ }^{-2}\right]_{0}\left[\mathrm{R}_{6}{ }^{2} \mathrm{R}_{6}{ }^{-2}\right]_{120}\left[\mathrm{R}_{6}{ }^{2} \mathrm{R}_{6}{ }^{-2}\right]_{240}
$$

where $[. .]_{\phi}$ represents an overall phase shift through the angle $\phi$. Some experimental results for $\mathrm{L}-\left[\mathrm{U}_{-}{ }^{13} \mathrm{C}\right]$-tyrosine are shown in Fig.3. 
Fig.4. Numerical simulations of powder-average Zeeman magnetization transfer between two ${ }^{13} \mathrm{C}$ spins, at a MAS frequency of $38 \mathrm{kHz}$. Top: the sequence in Eq.(3); Bottom: RFDR. The magnetization transfer is shown as a function of time (horizontal axis) and static field (specified as the proton Larmor frequency in $\mathrm{MHz}$ ). The simulation parameters correspond to $\left[\mathrm{U}-{ }^{13} \mathrm{C}\right]-$ glycine.

The preliminary numerical simulations shown in Fig.4 indicate that the supercycled $\mathrm{R}_{6}{ }^{2}$ sequence should display excellent performance at high magnetic fields (simulated up to $20 \mathrm{~T}$ ) and high sample spinning frequencies (simulated at $38 \mathrm{kHz}$ ), without requiring an excessively large rf field. Its predicted performance is more broadband with respect to chemical shifts and chemical shift anisotropies than RFDR, despite its lower rf field requirements. Furthermore, the new sequence also has much lower rf field requirements than the RIL method $[12,13]$, which normally cannot be implemented at all at high spinning frequencies. In addition, the sequence in Eq.(3) is expected to be tolerant to the setting of the rf phase shift. 
Fig5. (Top) Experimental ${ }^{13} \mathrm{C}$ spectrum of $\mathrm{L}-\left[{ }^{15} \mathrm{~N}, 2-{ }^{13} \mathrm{C}\right]$-alanine, obtained by taking the crosspolarized ${ }^{13} \mathrm{C}$ signal in the presence of a $\mathrm{R}_{18}{ }_{2}^{5}$ sequence at the ${ }^{1} \mathrm{H}$ Larmor frequency. The basic element of the $R 18_{2}{ }^{5}$ sequence was $R=180_{0}$. The static field was $9.4 T$, the spinning frequency was $18 \mathrm{kHz}$, and the proton nutation frequency was $81 \mathrm{kHz}$. (Bottom) Numerical simulation using a simple heteronuclear two-spin model, with an effective $\mathrm{CH}$ distance of $0.112 \mathrm{~nm}$.

Heteronuclear dipolar recoupling with homonuclear decoupling. The symmetry theorems also allow one to design sequences that recouple different spin species, such as ${ }^{13} \mathrm{C}$ and ${ }^{1} \mathrm{H}$ spins, at the same time as decoupling homonuclear spins from each other [7]. Fig. 5 shows the Fourier transform of ${ }^{13} \mathrm{C}$ signals acquired in the presence of $\mathrm{R} 18_{2}{ }^{5}$ irradiation of the protons. The $\mathrm{R} 18_{2}{ }^{5}$ sequence recouples the heteronuclear dipole-dipole interaction while decoupling the protons from each other. The spectral splitting is due to the recoupled heteronuclear dipole-dipole interaction between the ${ }^{13} \mathrm{C}$ spin and its directly-bonded ${ }^{1} \mathrm{H}$ neighbour. We anticipate that bond lengths and other internuclear distances may simply be read off such one-dimensional spectra, after calibration of the scaling factor $\kappa$. This will be useful for investigating hydrogen bonding and other structural issues. 
Other symmetries. A variety of symmetries for other decoupling tasks was listed in ref.[7]. We are also preparing a publication on multiple-channel rf sequences (A. Brinkmann, unpublished). An application of C-type sequences to the MAS of oriented systems is sketched by C. Glaubitz elsewhere in this volume.

Existing pulse sequences. The symmetry theorems sketched here also provide insight into the operation of a variety of well-known pulse sequences. For example, the REDOR scheme with xy phase cycling [14-16] conforms to $\mathrm{R} 4{ }_{2}{ }^{1}$ symmetry. This symmetry accounts for the good performance of this sequence, particularly in regimes for which it was not originally designed, such as at fast MAS frequencies [17]. However, the $\mathrm{R}_{2}{ }^{1}$ symmetry of REDOR also recouples the irradiated spins with each other -- this undesirable feature may often be unimportant when REDOR is applied to low- $\gamma$ spins such as ${ }^{15} \mathrm{~N}$, but should be of concern in other situations.

The RFDR homonuclear recoupling method [11], with xy phase cycling, conforms to $\mathrm{R} 4{ }_{4}{ }^{1}$ symmetry. This is an appropriate symmetry for the task of zero-quantum homonuclear recoupling [7]. However, the scaling factor $\kappa$ of RFDR vanishes in the limit of infinite rf field. The RFDR sequence only works at all because the duration of the rf pulses is finite, and because of assistance from higher-order interference terms involving the chemical shifts. As a result, the magnetization transfer under RFDR is strongly dependent on both the isotropic and anisotropic chemical shifts, as well as the rf field strength. The new symmetry-based sequences, such as the one given in Eq.(3), are expected to be more quantitative and reliable.

The successful TPPM heteronuclear decoupling method [18] is also amenable to symmetry analysis $[4,7]$.

\section{Conclusions}

The symmetry theorems described here allow a wide variety of decoupling and recoupling problems in solid-state NMR to be addressed in a rational and general way. Symmetry does not itself solve all problems, but does provide a sound starting point for designing pulse sequences. We have obtained promising results for double-quantum and zero-quantum homonuclear recoupling sequences, and for heteronuclear recoupling sequences. Some of these solutions are predicted to have good performance at very high magnetic fields and at very fast sample rotation frequencies. Other solutions provide robust and quantitative performance under moderate fields and spinning frequencies, in the presence of large chemical shift anisotropies.

My guess (M.H.L.) is that the future development of solid-state NMR in biology will continue to include several parallel strands, with high and low magnetic fields, high and low spinning frequencies, and a variety of labelling strategies, all playing important roles in different situations. In many cases very high static magnetic fields will lead to good spectral resolution, but these advantages should be balanced against the enhanced effects of the chemical shift anisotropies, which will often compromise the quantitation 
of methods for geometry determination. Similarly, the use of heavily-labelled compounds increases the information output per sample, but also degrades the quality and reliability of the geometrical information, and increases the complexity of the analysis. For these reasons, solid-state NMR on selectively-labelled compounds at moderate fields will continue to be a sound strategy for obtaining reliable and quantitative geometrical data, at least in many contexts. The biological solid-state NMR spectroscopists of the future will be faced with some interesting strategic choices.

In any case, the design of pulse sequences using symmetry, as sketched here, is versatile enough for a wide range of future scenarios. 


\section{Acknowledgements}

This research was supported by the Swedish Natural Science Research Council, the Göran Gustafsson Foundation for Research in the Natural Sciences and Medicine, and the European Union Marie Curie Fellowship Program. We would like to thank H.

Luthman for synthesis of the labelled retinals, A. Sebald for the loan of other labelled compounds, and Ole Johannessen for experimental help.

\section{References}

1. Lee, Y.K., Kurur, N.D., Helmle, M., Johannessen, O.G., Nielsen, N.C. and Levitt, M.H., Chem. Phys. Lett. 242 (1995) 304.

2. Hohwy, M., Jakobsen, H.J., Edén, M., Levitt, M.H. and Nielsen, N.C., J. Chem. Phys. 108 (1998) 2686.

3. Rienstra, C.M., Hatcher, M.E., Mueller, L.J., Sun, B., Fesik, S.W. and Griffin, R.G., J. Am. Chem. Soc. 120 (1998) 10602.

4. Edén, M. and Levitt, M.H., J. Chem. Phys. 111 (1999) 1511.

5. Hohwy, M., Rienstra, C.M., Jaroniec, C.P. and Griffin, R.G., J. Chem. Phys. 110 (1999) 7983.

6. Brinkmann, A., Edén, M. and Levitt, M.H., J. Chem. Phys. 112 (2000) 8539.

7. Carravetta, M., Edén, M., Zhao, X., Brinkmann, A. and Levitt, M.H., Chem. Phys. Lett. 321 (2000) 205.

8. Tycko, R. and Dabbagh, G., J. Am. Chem. Soc. 113 (1991) 9444.

9. Nielsen, N.C., Bildsøe, H., Jakobsen, H.J. and Levitt, M.H., J. Chem. Phys. 101 (1994) 1805.

10. Hamanaka, T., Mitsui, T., Ashida, T. and Kakudo, M., Acta Cryst. B28 (1972) 214.

11. Bennett, A.E., Ok, J.H., Griffin, R.G. and Vega, S., J. Chem. Phys. 96 (1992) 8624.

12. Baldus, M., Tomaselli, M., Meier, B.H. and Ernst, R.R., Chem. Phys. Lett. 230 (1994) 329.

13. Baldus, M., Geurts, D.G. and Meier, B.H.,

Sol. State Nucl. Magn. Reson. 11 (1998) 157.

14. Gullion, T. and Schaeffer, J., J. Magn. Reson. 81 (1989) 196.

15. Garbow, J.R. and Gullion, T., J. Magn. Reson. 95 (1991) 442.

16. Holl, S.M., Marshall, G.R., Beusen, D.D., Kociolek, K., Redlinski, A.S., Leplawy, M.T., McKay, R.A., Vega, S. and Schaefer, J., J. Am. Chem. Soc. 114 (1992) 4830.

17. Jaroniec, C.P., Tounge, B.A., Rienstra, C.M., Herzfeld, J. and Griffin, R.G., J. Magn. Reson. 146 (2000) 132.

18. Bennett, A.E., Rienstra, C.M., Auger, M., Lakshmi, K.V. and Griffin, R.G., J. Chem. Phys. 103 (1995) 1. 


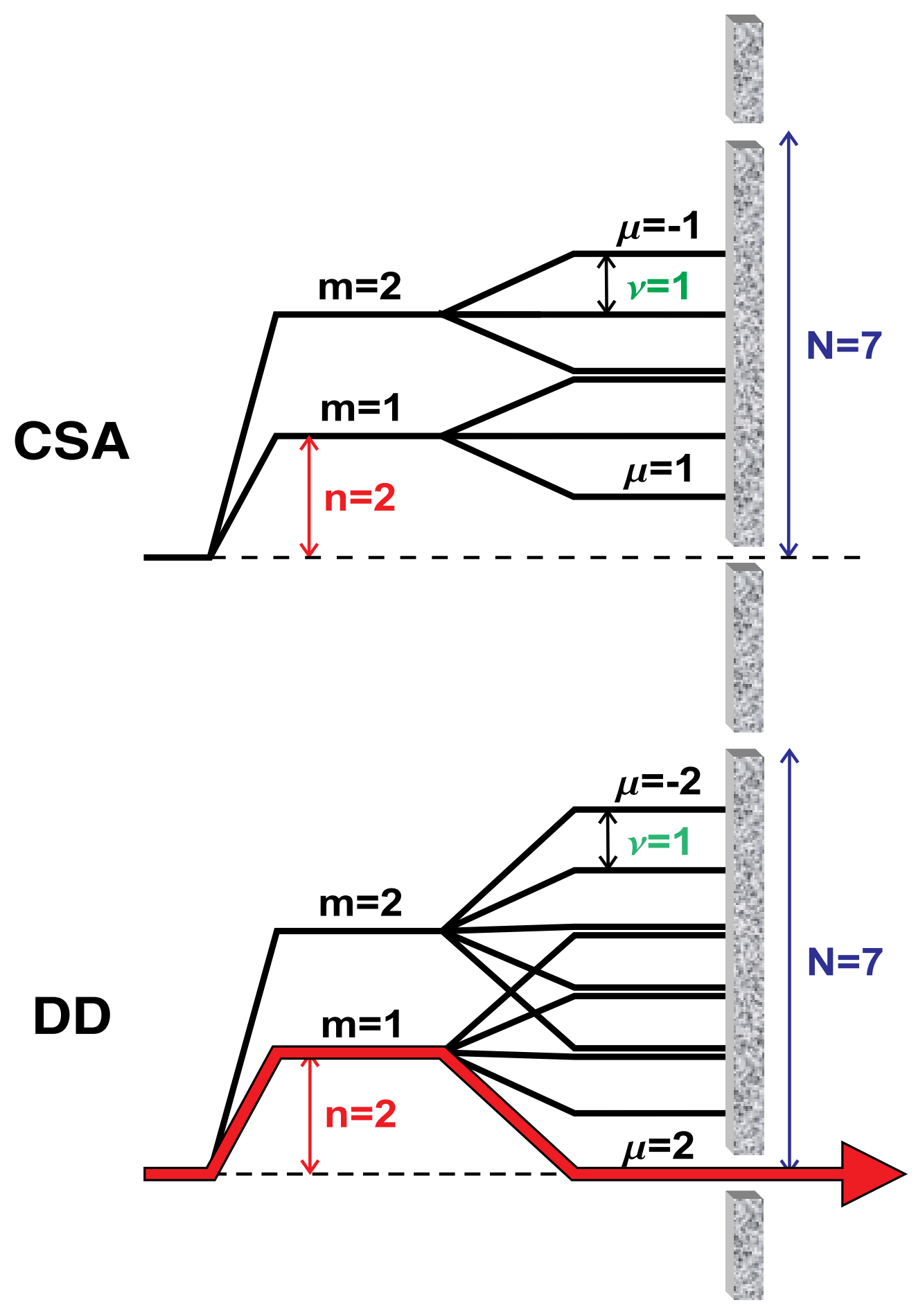

Brinkmann et al. Fig.1 


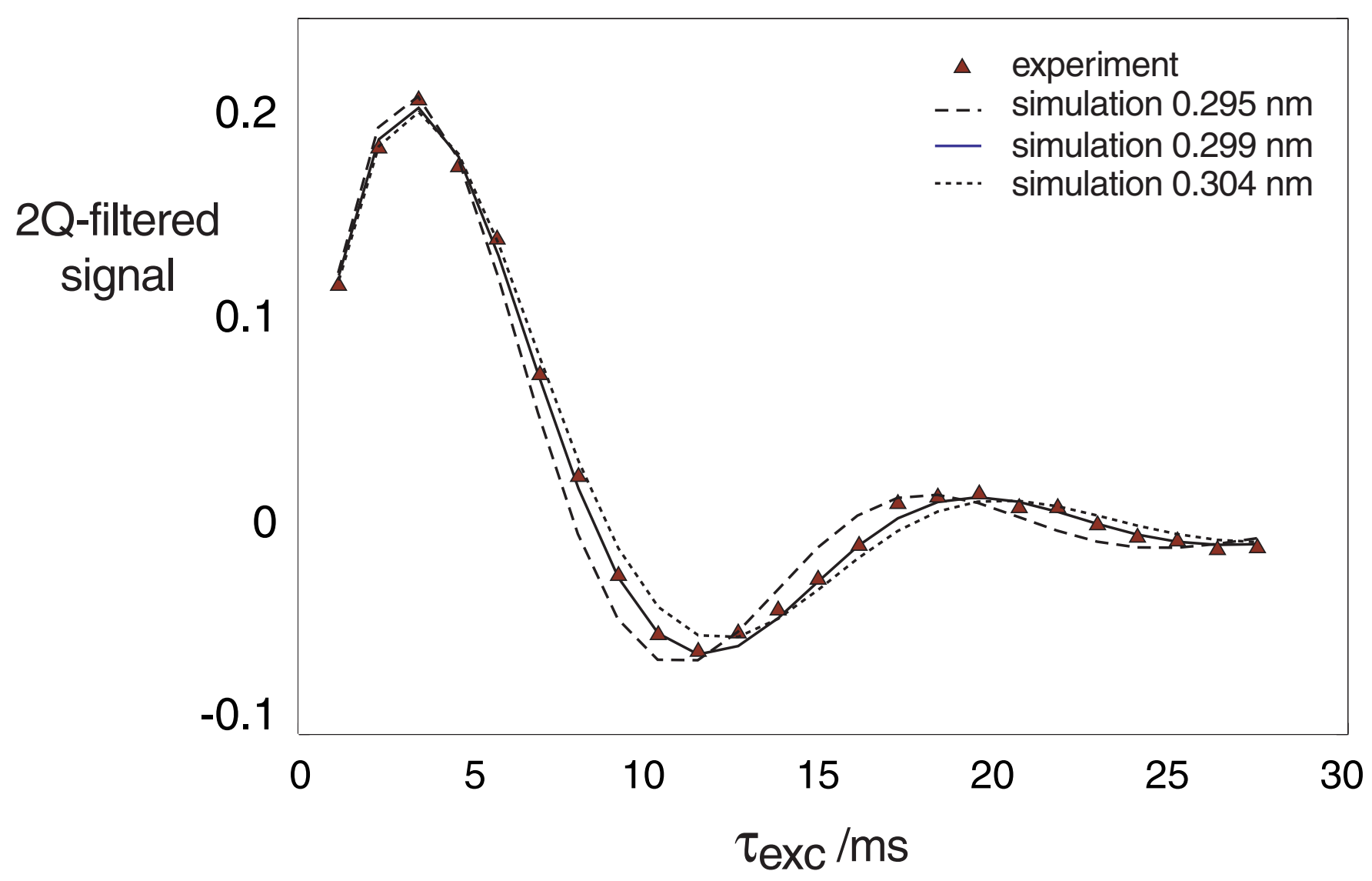

Brinkmann et al. Fig.2 


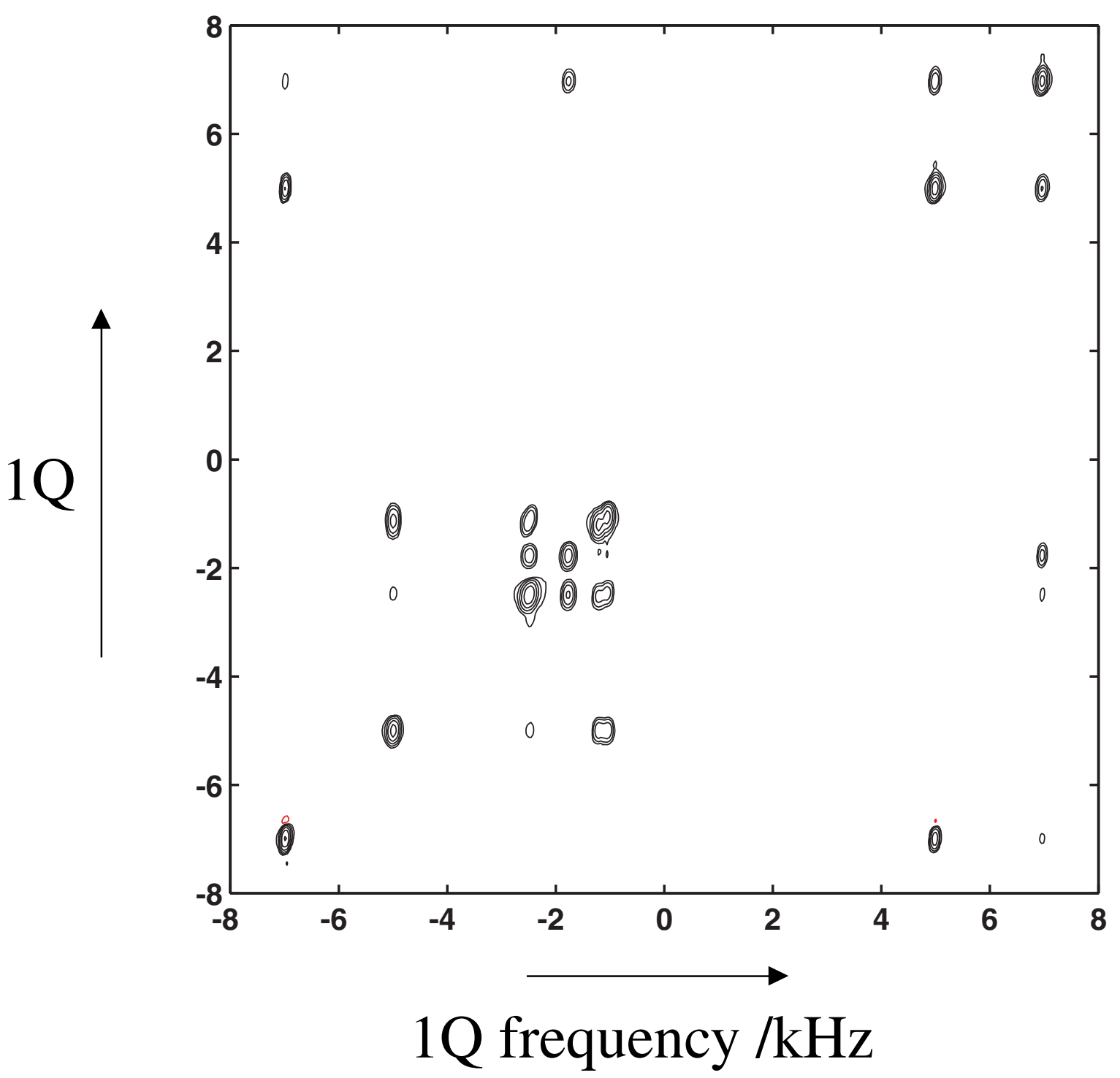

Brinkmann et al. Fig.3 


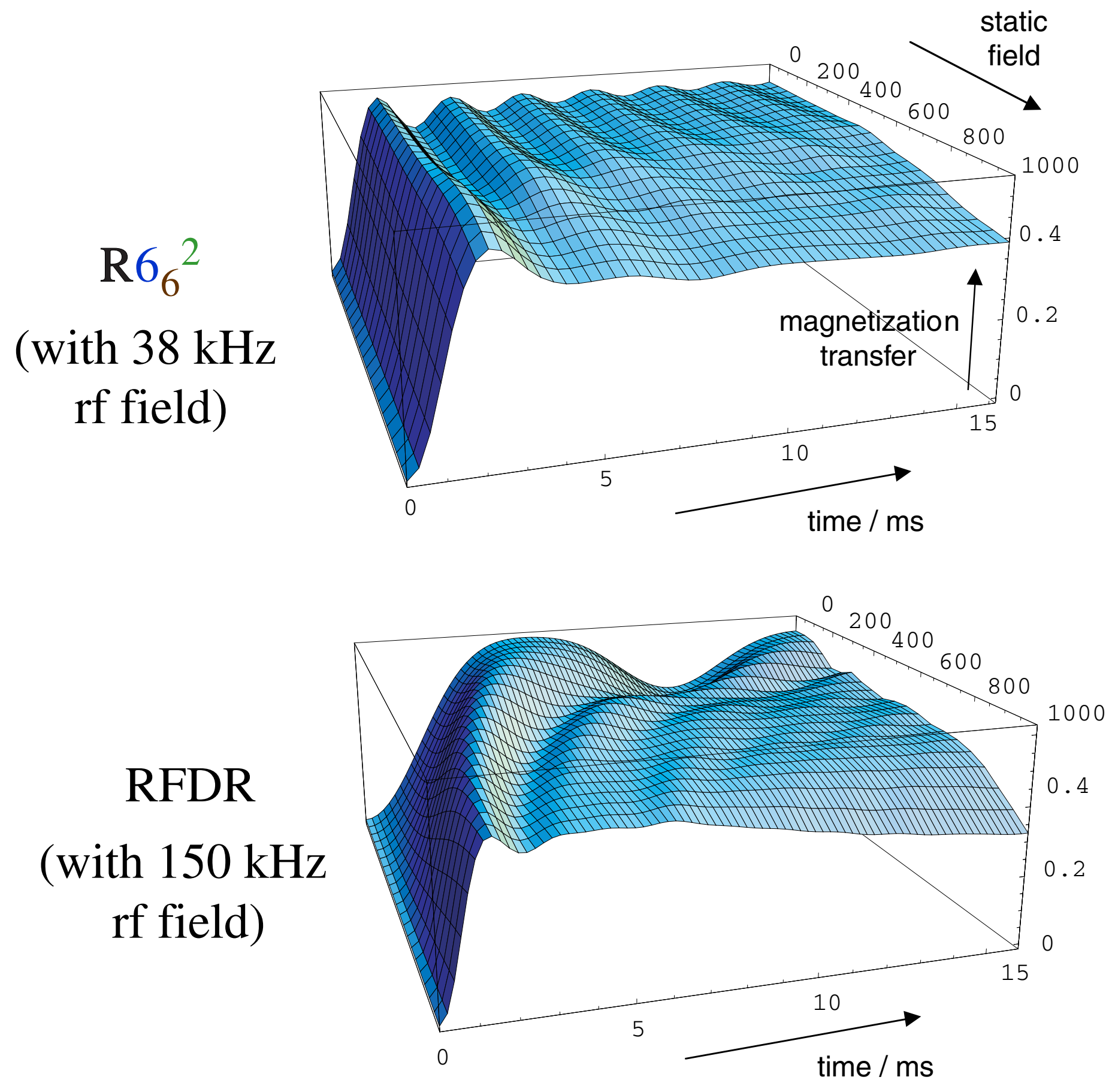

Brinkmann et al. Fig.4 


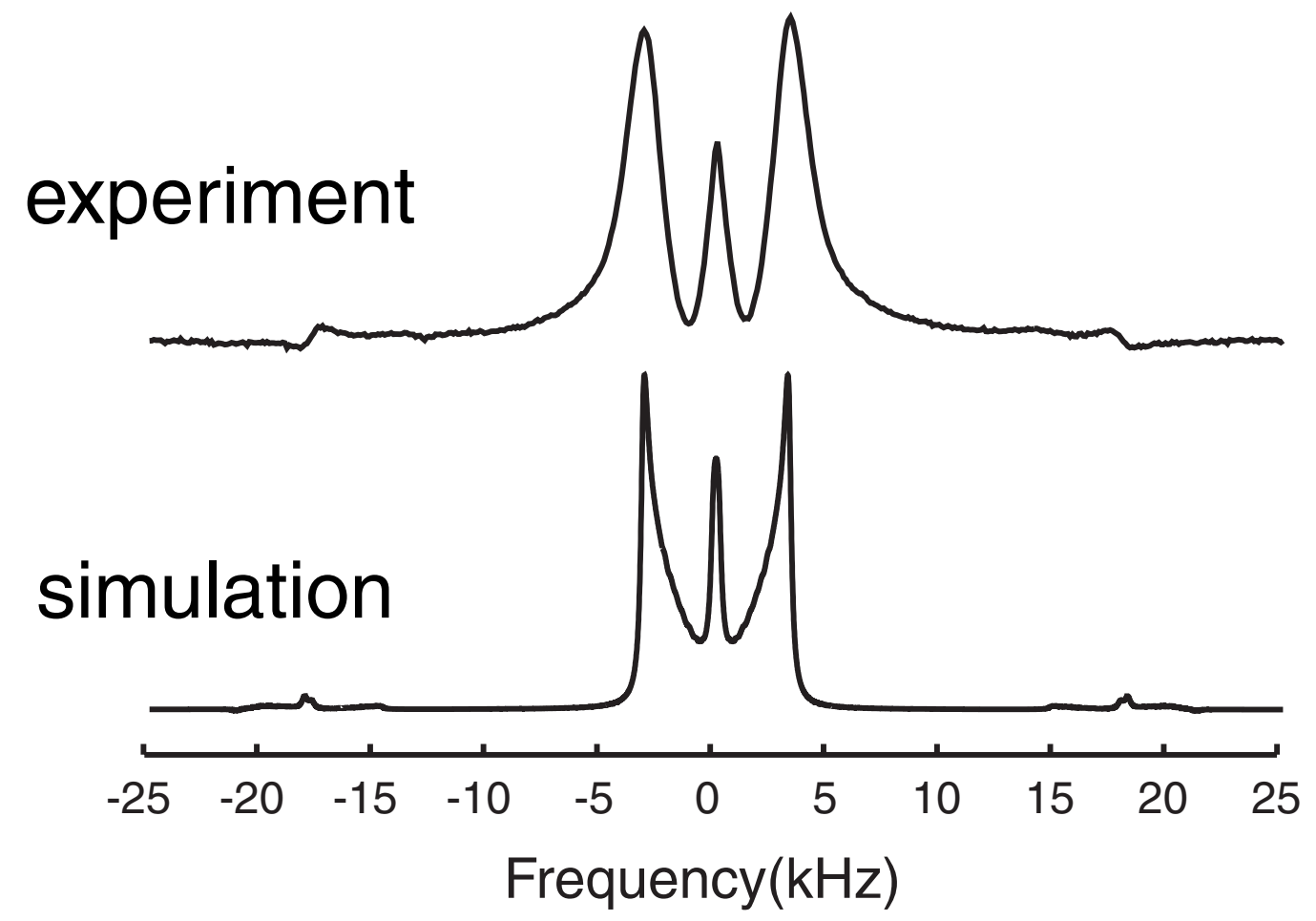

Brinkmann et al. Fig.5 